\title{
Diet quality, nutrition and physical activity among adolescents: the Web-SPAN (Web-Survey of Physical Activity and Nutrition) project
}

\author{
Kate E Storey ${ }^{1, *}$, Laura E Forbes ${ }^{1}$, Shawn N Fraser ${ }^{2}$, John C Spence ${ }^{3}$, \\ Ronald C Plotnikoff ${ }^{3,4}$, Kim D Raine ${ }^{4}$, Rhona M Hanning ${ }^{5}$ and Linda J McCargar ${ }^{1}$ \\ ${ }^{1}$ Alberta Institute for Human Nutrition, Department of Agricultural, Food and Nutritional Science, 4-10 \\ Agriculture/Forestry Centre, University of Alberta, Edmonton, Alberta, Canada, T6G 2P5: ${ }^{2}$ Centre for Nursing \\ and Health Studies, Athabasca University, Athabasca, Alberta, Canada: ${ }^{3}$ Faculty of Physical Education \\ and Recreation, University of Alberta, Edmonton, Alberta, Canada: ${ }^{4}$ Centre for Health Promotion Studies, \\ School of Public Health, University of Alberta, Edmonton, Alberta, Canada: ${ }^{5}$ Department of Health Studies \\ and Gerontology, University of Waterloo, Waterloo, Ontario, Canada
}

Submitted 25 August 2008: Accepted 29 April 2009: First published online 23 June 2009

\begin{abstract}
Objective: To assess the overall diet quality of a sample of adolescents living in Alberta, Canada, and evaluate whether diet quality, nutrient intakes, meal behaviours (i.e. meal skipping and consuming meals away from home) and physical activity are related.

Design: A cross-sectional study design. Students completed the self-administered Web-Survey of Physical Activity and Nutrition (Web-SPAN). Students were classified as having poor, average or superior diet quality based on Canada's Food Guide to Healthy Eating (CFGHE).

Setting: One hundred and thirty-six schools (37\%) within forty-four public and private school boards (75\%) in Alberta, Canada.

Subjects: Grade 7 to 10 Alberta students ( $n$ 4936) participated in the school-based research.

Results: On average, students met macronutrient requirements; however, micronutrient and fibre intakes were suboptimal. Median CFGHE food group intakes were below recommendations. Those with poor diet quality (42\%) had lower intakes of protein, fibre and low-calorie beverages; higher intakes of carbohydrates, fat and Other Foods (e.g. foods containing mostly sugar, high-salt/fat foods, high-calorie beverages, low-calorie beverages and high-sugar/fat foods); a lower frequency of consuming breakfast and a higher frequency of consuming meals away from home; and a lower level of physical activity when compared with students with either average or superior diet quality.

Conclusions: Alberta adolescents were not meeting minimum CFGHE recommendations, and thus had suboptimal intakes and poor diet quality. Suboptimal nutritional intakes, meal behaviours and physical inactivity were all related to poor diet quality and reflect the need to target these health behaviours in order to improve diet quality and overall health and wellness.
\end{abstract}

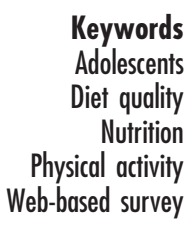

Recent national surveys reveal Canadian adolescents have suboptimal nutritional intakes and low levels of physical activity $^{(1-3)}$. Although these results provide insight into the current health behaviours of Canadian adolescents, less is known about the diet quality of this population judged in terms of adherence to recommendations established by Canada's Food Guide to Healthy Eating (CFGHE), the national nutrition guidance in Canada at the time of data collection (CFGHE was recently revised in 2007). Although each food group is individually important, total health and wellness relies on the appropriate balance and variety of all four food groups ${ }^{(4)}$ as well as adequate levels of physical activity ${ }^{(5)}$. Further, the relationship between diet quality and nutrient intakes, meal behaviours (i.e. meal skipping and consuming meals away from home) and physical activity is not well understood as few studies have examined the co-occurrence of these behaviours in adolescents $^{(6)}$

Unhealthy diets and physical inactivity have been established as important determinants of the rising prevalence 
of overweight and obesity among children and adolescents $^{(1,7)}$. Due to significant long-term health and economic implications of adolescent obesity, research in the area of obesity determinants has become increasingly important. Specifically, diet quality has become a useful composite score for comparing dietary intake with other risk factors, and has been linked to meal skipping ${ }^{(8)}$, nutrient inadequacy and higher percentage of body/ abdominal fat ${ }^{(9)}$.

Because adolescents are at a life stage where they have increased autonomy regarding health behaviours, many of the decisions and choices adolescents make may persist into adulthood and have a lasting impact on long-term health ${ }^{(10)}$. Therefore, the purpose of the present study was to assess overall diet quality and physical activity levels of a sample of Alberta adolescents, and evaluate the relationship between diet quality, nutrient intakes, meal behaviours (i.e. meal skipping and consuming meals away from home) and physical activity levels.

\section{Experimental methods}

\section{Participants}

The Web-Survey of Physical Activity and Nutrition (WebSPAN) was a self-administered web-based survey of grade 7 to 10 students that assessed diet, physical activity and related meal behaviours (i.e. meal skipping and consuming meals away from home). All fifty-nine public and separate school boards in Alberta, Canada were selected for participation and forty-eight school boards agreed to participate (81\%). In order to maximize school recruitment and reduce the impact of cluster randomization caused by the lack of variability between students within school boards and schools, an average of seven schools (the average maximum number of eligible schools) within each school board were randomly chosen and contacted after obtaining school board approval. Private schools were randomly selected based on regional diversity. All students within the selected classrooms were invited to participate.

In total, 363 schools were contacted of which 193 (53\%) agreed to participate, 160 (44\%) declined participation and ten $(3 \%)$ were ineligible based on inappropriate grade levels. Upon study completion, final participation was 136 (37\%) schools within forty-four (75\%) school boards. In total, 18484 students were contacted and 5848 (32\%) students provided parental consent. However, due to school time constraints, only 4981 adolescents participated in the study. We excluded participating students who signed on to the website but did not complete any part of the survey ( $n 9)$ and students with extreme values of total energy intake or physical activity levels based on outlier analyses using the SPSS statistical software package version $15 \cdot 0$ (SPSS Inc., Chicago, IL, USA; total energy intake, $n$ 24; physical activity, $n 14$; total outliers removed, $n 36$ ). Thus, a final sample of
$4936(27 \%)$ adolescents were included in the analyses ( 2264 boys; 2623 girls; mean age $=13.6$ years). Age and sex characteristics of our overall sample reflected the sampling frame of the provincial school population for grade $7-10$ adolescents $^{(11)}$. During data collection, students were free to end the survey at any time; therefore, sample size varies throughout the analyses.

\section{Measures}

\section{Dietary intake}

As part of Web-SPAN, a $24 \mathrm{~h}$ dietary recall was administered to students to measure weekday dietary intake using the web-based Food Behaviour Questionnaire (FBQ) developed at the University of Waterloo ${ }^{(12)}$ and modified by researchers at the University of Alberta by adding questions to assess meal behaviours (i.e. meal skipping and consuming meals away from home), selfefficacy and physical activity. Responding to electronic survey questions, students reported all foods and beverages consumed the previous day. In addition, a portion of the sample ( $n 580 ; 12 \%$ ) completed repeat 24 h dietary recalls using Web-SPAN to assess usual intake. To help students recall their intake, portion size images and cues regarding beverage intake were provided.

The $24 \mathrm{~h}$ dietary recall component of the FBQ has been validated using several methods. In comparison to a dietitian-administered $24 \mathrm{~h}$ dietary recall, the FBQ showed good agreement for total energy intake, macronutrients and key micronutrients in grade $6-8$ students $^{(13)}$. A subset of students who participated in the current study completed the survey on two days ( $n$ 379), and also completed a $3 \mathrm{~d}$ food record ( $n$ 369). Intra-class correlation coefficient (ICC) values for the repeat comparisons and between the FBQ and the $3 \mathrm{~d}$ food record were within ranges reported elsewhere in the adolescent population $^{(14)}$. Furthermore, mean differences of nutrient intakes between the two measurements were small ${ }^{(15)}$.

A measure of diet quality was used to classify students into groups of poor, average or superior diet quality using a food-based diet quality index ${ }^{(16)}$ modified to reflect $\mathrm{CFGHE}^{(4)}$ foods. As suggested by Waijers et al. ${ }^{(17)}$, foodbased indices are the preferred type of dietary index used for health promotion purposes and are useful when measuring the degree of adherence to dietary recommendations. In the current study, servings of each of the four food groups according to $\mathrm{CFGHE}^{(4)}$ were calculated using data from the $24 \mathrm{~h}$ dietary recall. Foods that were not classified according to CFGHE were categorized as Other Foods and further divided into sub-categories which included foods containing mostly sugar (e.g. candies), high-salt/fat foods (e.g. potato chips), high-calorie beverages (e.g. regular soft drinks), low-calorie beverages (e.g. low-calorie soft drinks) and high-sugar/fat foods (e.g. pastries) based on Canadian Nutrient File definitions ${ }^{(18)}$. Diet quality was defined using the number of 
minimum food group recommendations that were met based on the 1992 CFGHE. Individuals were classified as having either poor (met 0 or 1), average (met 2 or 3 ) or superior (met all 4) diet quality. Although not validated, the food groupings are similar to the tertiles reported by Lowik et $\mathrm{al}^{(16)}$ and were supported by graded responses in key nutrient intakes in each group, where improvements in nutrient intakes were observed when diet quality increased from poor to average to superior. CFGHE was recently revised and the new food guide ${ }^{(19)}$ was released in early 2007. In spite of this, the 1992 CFGHE was the government recommendation during data collection and was also a component of the Alberta school curriculum ${ }^{(20,21)}$. Therefore, it was most appropriate to compare intakes with the 1992 CFGHE recommendations.

Macronutrient and micronutrient intakes were assessed using ESHA Food Processor software version 7.9 (ESHA Research, Salem, OR, USA) and the 2001b Canadian Nutrient File ${ }^{(18)}$ database and compared with the Dietary Reference Intakes (DRI) ${ }^{(22-26)}$. Estimates of inadequacy were calculated for nutrients with an Estimated Average Requirement $(\mathrm{EAR})^{(27)}$. Inadequacy was not determined for nutrients with an Adequate Intake (AI), as many values below the AI are likely to be adequate ${ }^{(27)}$. Macronutrients were compared with the Acceptable Macronutrient Distribution Ranges (AMDR).

\section{Meal behaviours}

Survey questions examining meal behaviours (i.e. meal skipping and consuming meals away from home) are comparable to questions developed for Project EAT (Eating Among Teens), a well-established survey instrument ${ }^{(28,29)}$. Frequency of meal consumption was assessed by asking 'How often do you usually eat...?' followed by 'breakfast', 'lunch', 'dinner', 'morning snacks', 'afternoon snacks' or 'evening snacks', with response options of 'never', 'on weekends only', 'less than half of the week (three or fewer days each week)', 'more than half of the week (four or more days each week)', 'every day' and 'not answered'. Frequency of consuming meals away from home was assessed by asking 'How often do you eat meals or snacks prepared away from home?', with response options of 'rarely or never', 'once a month', 'once a week', '2-6 times a week' and 'once a day'. The following locations were assessed: school cafeteria, fastfood restaurant or take out, other restaurants, vending machines, snack bars and convenience stores.

\section{Physical activity}

A web-based version of the Physical Activity Questionnaire for Older Children (PAQ-C) ${ }^{(30)}$ was used to assess physical activity levels over a $7 \mathrm{~d}$ period. The PAQ-C has been found to be a reliable ${ }^{(31)}$ and valid measure ${ }^{(32)}$ in adolescents. In addition, among a subset of the sample ( $n$ 323), we observed good test-retest reliability for the web-based PAQ-C completed approximately $8 \mathrm{~d}$ apart $(r=0 \cdot 79)$.
Further, when compared with the guided self-administered paper-based PAQ-C, the web-based PAQ-C showed good agreement $(\mathrm{ICC}=0 \cdot 70 \text {; Pearson } r=0 \cdot 70 ; n 411)^{(15)}$.

\section{Procedures}

School boards and schools were individually contacted by mail and follow-up telephone calls were made to request permission to survey students. Parents and students received information letters, and parental consent was obtained using active consent. All students provided assent after signing on to the web-based survey. The anonymous 24-page survey took approximately $45 \mathrm{~min}$ to complete and was conducted during class time within the school day. Survey data were collected between January and November 2005 (except in July and August when schools were closed for summer) and thus nutritional intakes may have been affected by seasonal variation. This research was approved by a university ethics board and by all participating school boards and schools.

\section{Statistical analyses}

Macronutrient intakes, food group intakes, physical activity levels and differences in intake and physical activity levels between sex and age were analysed using descriptive statistics, $\chi^{2}$ tests, $t$ tests and Mann-Whitney $U$ tests. ANOVA was used to assess the difference in physical activity levels between those with poor, average and superior diet quality. In order to assess the prevalence of micronutrient inadequacy (expressed as the percentage below the EAR), two days of intake data were used to adjust for intra-individual variation ${ }^{(33)}$ using the Software for Intake Distribution Estimation (PC-SIDE) ${ }^{(34)}$.

A $2 \times 3$ multivariate analysis of covariance (MANCOVA), with total energy intake as the covariate, was used to evaluate the association between sex and total diet quality. Univariate follow-ups were completed on significant MANCOVA results for dependent variables including nutrient intakes, servings of Other Foods, and frequency of meal consumption and consuming meals away from home. Prior to MANCOVA analyses, the assumption of homogeneous slopes $^{(35,36)}$ was tested, and interactions indicated that the slopes differed among levels of the independent variables. Therefore, adjusted values were based on custom models which included these interactions for the following MANCOVA analyses: macronutrients, Other Foods and frequency of meal consumption.

Statistical analyses were performed using SPSS version $15 \cdot 0$. Due to the large sample size, a criterion $\alpha$ level of $P<0 \cdot 01$ was used for all statistical comparisons.

\section{Results}

\section{Nutrient intakes}

Results of the $24 \mathrm{~h}$ recall data revealed, on average, that the distribution of energy from macronutrients was within 
the AMDR for both boys $(53.4 \%$ carbohydrate, $15 \cdot 8 \%$ protein, $32 \cdot 2 \%$ fat) and girls ( $55 \cdot 8 \%$ carbohydrate, $14 \cdot 9 \%$ protein, $31 \cdot 0 \%$ fat). Boys consumed significantly more total energy (boys, 10033 (sD 5038) kJ/d (2398 (sD 1204) $\mathrm{kcal} / \mathrm{d}$ ); girls, 7535 (sD 3820) kJ/d (1801 (sD 913) kcal/d)) and percentage of energy from protein (boys, $15 \cdot 8$ (SD 5.4 ) \%; girls, $14 \cdot 9$ (SD 5.2) \%) and fat (boys, $32 \cdot 2$ (SD 9.1) $\%$; girls, $31 \cdot 0$ (sD 9.2) \%) than girls; while girls consumed significantly more energy from carbohydrate (boys, 53.4 (sD 11.4) \%; girls, 55.8 (sD 11.2) \%) than boys $(P<0.001$ for energy and all macronutrients).

Adjusted average nutrient intakes and prevalence of micronutrient inadequacy are presented in Table 1. CFGHE data are presented as median food group intakes and interquartile ranges with comparisons based on sex; significant differences were observed between boys and girls for all food groups (Table 2).

\section{Diet quality}

Overall diet quality indicated that $42 \%$ ( $n$ 2020) of students had poor diet quality, 50\% ( $n$ 2450) had average diet quality and $8 \%(n$ 397) had superior diet quality. Among boys, 32\% ( $n$ 721), 56\% ( $n$ 1263) and 12\% ( $n$ 268) had poor, average and superior diet quality, respectively. In comparison, $50 \%$ ( $n$ 1299), $45 \%$ ( $n$ 1187) and $5 \%(n 129)$ of girls had poor, average and superior diet quality, respectively.

\section{Diet quality and nutrient intakes}

Multivariate analysis used to examine factors associated with macronutrient intakes revealed significant $F$ values for diet quality (Wilks' $\lambda=0 \cdot 96, \quad F[8,9708]=22 \cdot 51$, $P<0 \cdot 001, \eta_{\mathrm{p}}^{2}=0 \cdot 018$ ), while sex (Wilks' $\lambda=1 \cdot 00, F[4$, $\left.4854]=2 \cdot 38, P=0.049, \eta_{\mathrm{p}}^{2}=0.002\right)$ and the interaction between diet quality and sex were not significant (Wilks' $\left.\lambda=1 \cdot 00, \quad F[8,9708]=1 \cdot 13, P=0 \cdot 340, \eta_{\mathrm{p}}^{2}=0 \cdot 001\right)$. Univariate $F$ values for the main effect of diet quality indicated those with poor diet quality had significantly lower intakes of protein and fibre, and significantly higher intakes of carbohydrates and fat, compared with those with average and/or superior diet quality (Table 3 ).

The association between diet quality and sex with Other Foods sub-categories (e.g. foods containing mostly sugar, high-salt/fat foods, high-calorie beverages, lowcalorie beverages and high-sugar/fat foods) as assessed using MANCOVA revealed significant $F$ values for sex (Wilks' $\left.\lambda=1 \cdot 00, F[5,4853]=4 \cdot 79, P<0 \cdot 001, \eta_{\mathrm{p}}^{2}=0 \cdot 005\right)$ and diet quality (Wilks' $\lambda=0.99, F[10,9706]=6.45$, $\left.P<0.001, \eta_{\mathrm{p}}^{2}=0.007\right)$ and the interaction between diet quality and sex (Wilks' $\lambda=1 \cdot 00, \quad F[10,9706]=2 \cdot 45$, $\left.P=0 \cdot 006, \eta_{\mathrm{p}}^{2}=0 \cdot 003\right)$. Interactions indicated that boys in the poor diet quality group consumed more high-calorie beverages than girls in the same group. Boys consumed less low-calorie beverages $(F[1,4857]=15 \cdot 17, P<0 \cdot 001$, $\left.\eta_{\mathrm{p}}^{2}=0.003\right)$ than girls. Univariate analyses for the main effect of diet quality showed significant $F$ values for all
Table $1 \mathrm{DRI}^{(22-26)}$ and adjusted Al compared with recommendations among adolescents aged $11-17$ years ${ }^{*}$, Alberta, Canada

\begin{tabular}{|c|c|c|c|}
\hline Nutrient & $\begin{array}{c}\text { DRI value } \\
\text { (DRI category) }\end{array}$ & $\begin{array}{c}\text { Adjusted } \\
\text { Alt }\end{array}$ & $\begin{array}{c}\text { Percentage } \\
\text { below the EAR }\end{array}$ \\
\hline \multicolumn{4}{|l|}{ Vitamin A $(\mu \mathrm{g}) \S$} \\
\hline Boys $9-13$ & 445 (EAR) & $22 \cdot 0$ & \\
\hline Boys $14-17$ & 630 (EAR) & $23 \cdot 9$ & \\
\hline Girls 9-13 & 420 (EAR) & $23 \cdot 7$ & \\
\hline Girls $14-17$ & 485 (EAR) & $22 \cdot 9$ & \\
\hline \multicolumn{4}{|l|}{ Vitamin C (mg) } \\
\hline Boys $9-13$ & 39 (EAR) & $133 \cdot 4$ & $6 \cdot 4$ \\
\hline Boys $14-17$ & 63 (EAR) & $137 \cdot 3$ & $18 \cdot 2$ \\
\hline Girls 9-13 & 39 (EAR) & $134 \cdot 6$ & $6 \cdot 7$ \\
\hline Girls $14-17$ & 56 (EAR) & $126 \cdot 0$ & $12 \cdot 1$ \\
\hline \multicolumn{4}{|l|}{$\mathrm{Fe}(\mathrm{mg}) \|$} \\
\hline Boys $9-13$ & 5.9 (EAR) & $14 \cdot 1$ & $2 \cdot 6$ \\
\hline Boys $14-17$ & $7 \cdot 7$ (EAR) & $16 \cdot 1$ & $3 \cdot 9$ \\
\hline Girls 9-13 & $5 \cdot 7$ (EAR) & $10 \cdot 6$ & $6 \cdot 4$ \\
\hline Girls 14-17 & $7 \cdot 9$ (EAR) & $10 \cdot 5$ & $21 \cdot 5$ \\
\hline \multicolumn{4}{|l|}{ Niacin (mg) } \\
\hline Boys $9-13$ & 9 (EAR) & $19 \cdot 4$ & $4 \cdot 0$ \\
\hline Boys $14-17$ & 12 (EAR) & $20 \cdot 8$ & $10 \cdot 2$ \\
\hline Girls 9-13 & 9 (EAR) & $14 \cdot 7$ & $13 \cdot 2$ \\
\hline Girls $14-17$ & 11 (EAR) & $14 \cdot 7$ & $21 \cdot 2$ \\
\hline \multicolumn{4}{|l|}{$\mathrm{Zn}(\mathrm{mg})$} \\
\hline Boys 9-13 & $7 \cdot 0$ (EAR) & $10 \cdot 9$ & $20 \cdot 3$ \\
\hline Boys $14-17$ & 8.5 (EAR) & $11 \cdot 9$ & $19 \cdot 6$ \\
\hline Girls 9-13 & $7 \cdot 0$ (EAR) & $8 \cdot 1$ & $37 \cdot 7$ \\
\hline Girls $14-17$ & $7 \cdot 3$ (EAR) & $7 \cdot 7$ & $49 \cdot 2$ \\
\hline \multicolumn{4}{|l|}{$\mathrm{Ca}(\mathrm{mg})$} \\
\hline Boys $9-13$ & $1300(\mathrm{Al})$ & $1132 \cdot 9$ & \\
\hline Boys $14-17$ & 1300 (AI) & $1197 \cdot 5$ & \\
\hline Girls $9-13$ & $1300(\mathrm{Al})$ & $945 \cdot 6$ & \\
\hline Girls 14-17 & $1300(\mathrm{Al})$ & $910 \cdot 1$ & \\
\hline \multicolumn{4}{|l|}{ Vitamin D $(\mu \mathrm{g})$} \\
\hline Boys $9-13$ & $5(\mathrm{Al})$ & $5 \cdot 7$ & \\
\hline Boys $14-17$ & $5(\mathrm{Al})$ & $5 \cdot 7$ & \\
\hline Girls 9-13 & $5(\mathrm{Al})$ & $4 \cdot 4$ & \\
\hline Girls 14-17 & $5(\mathrm{Al})$ & $4 \cdot 2$ & \\
\hline \multicolumn{4}{|l|}{ Fibre $(\mathrm{g})$} \\
\hline Boys 9-13 & $31(\mathrm{Al})$ & $15 \cdot 3$ & \\
\hline Boys $14-17$ & $38(\mathrm{Al})$ & $16 \cdot 4$ & \\
\hline Girls 9-13 & $26(\mathrm{Al})$ & $13 \cdot 6$ & \\
\hline Girls 14-17 & $26(\mathrm{Al})$ & $13 \cdot 1$ & \\
\hline
\end{tabular}

DRI, Dietary Reference Intake; Al, Adequate Intake; EAR, Estimated Average Requirement.

${ }^{*}$ Four students were younger than age 11 years due to mixed grade level classrooms and were included in the analysis.

tAdjusted for intra-individual variation of intake using the Software for Intake Distribution Estimation (PC-SIDE).

‡Calculated only for nutrients with an EAR as recommended by the Institute of Medicine ${ }^{(27)}$.

\$An estimate of inadequacy (percentage below the EAR) could not be determined for vitamin $A$ due to high frequencies of individual intakes at $0 \mu \mathrm{g}$ ( $n$ 3880).

\|Although the EAR for $\mathrm{Fe}$ was established to replace losses, there is considerable variation in the losses that occur during menstruation. The resulting effect is that the percentage below the EAR determined using the EAR cut-point method likely underestimated the true prevalence of inadequacy ${ }^{(24)}$.

Other Foods sub-categories. Those with poor diet quality had significantly higher intakes of foods containing mostly sugar, high-salt/fat foods, high-calorie beverages and high-sugar/fat foods compared with both those with average diet quality as well as those with superior diet quality; while individuals with poor diet quality had significantly lower intakes of low-calorie beverages compared with those with superior diet quality (Table 3). 
Table 2 CFGHE recommendations ${ }^{(4)}$ and interquartile ranges of servings consumed by adolescents aged 11-17 years*, Alberta, Canada

\begin{tabular}{|c|c|c|c|c|c|c|c|}
\hline \multirow[b]{3}{*}{ CFGHE group (recommended daily servings) } & \multicolumn{6}{|c|}{ Number of servings } & \multirow[b]{3}{*}{$P$ valuet } \\
\hline & \multicolumn{3}{|c|}{ Boys ( $n$ 2252) } & \multicolumn{3}{|c|}{ Girls (n 2615) } & \\
\hline & 25th & 50th & 75th & 25th & 50th & 75th & \\
\hline Grain Products (5-12) & $3 \cdot 3$ & $5 \cdot 6$ & $8 \cdot 8$ & $2 \cdot 2$ & $4 \cdot 0$ & $6 \cdot 4$ & $<0.001$ \\
\hline Vegetables and Fruit $(5-10)$ & $2 \cdot 2$ & $4 \cdot 4$ & $7 \cdot 4$ & $2 \cdot 2$ & $4 \cdot 1$ & $6 \cdot 5$ & 0.005 \\
\hline Milk Products (10-16 years: $3-4)$ & 0.9 & $2 \cdot 3$ & 3.9 & $0 \cdot 6$ & $1 \cdot 7$ & $3 \cdot 1$ & $<0.001$ \\
\hline Meat and Alternatives (2-3) & $1 \cdot 5$ & $3 \cdot 0$ & $5 \cdot 0$ & $0 \cdot 8$ & $1 \cdot 9$ & $3 \cdot 3$ & $<0.001$ \\
\hline Other Foods (in moderation) & $3 \cdot 0$ & $5 \cdot 5$ & $9 \cdot 0$ & $2 \cdot 4$ & $4 \cdot 5$ & $7 \cdot 3$ & $<0.001$ \\
\hline
\end{tabular}

CFGHE, Canada's Food Guide to Healthy Eating.

${ }^{*}$ Four students were younger than age 11 years due to mixed grade level classrooms and were included in the analysis.

tSignificance of the difference between sexes using the Mann-Whitney $U$ test.

Table 3 Group differences in adjusted* nutrient intakes based on diet quality+ among adolescents aged 11-17 years, Alberta, Canada

\begin{tabular}{|c|c|c|c|c|c|c|c|c|}
\hline & \multicolumn{6}{|c|}{ Diet qualitył } & \multirow[b]{3}{*}{$F$ value } & \multirow[b]{3}{*}{$P$ value } \\
\hline & \multicolumn{2}{|c|}{ Poor } & \multicolumn{2}{|c|}{ Average } & \multicolumn{2}{|c|}{ Superior } & & \\
\hline & Mean & SE & Mean & SE & Mean & SE & & \\
\hline \multicolumn{9}{|l|}{ Nutrients (g) ( $n$ 4867) } \\
\hline Carbohydrate & $287 \cdot 45^{a}$ & $1 \cdot 82$ & $274 \cdot 86^{b}$ & $1 \cdot 15$ & $275 \cdot 48$ & $4 \cdot 43$ & $17 \cdot 26$ & $<0 \cdot 001^{\mathrm{ab}}$ \\
\hline Protein & $67 \cdot 66^{\mathrm{a}}$ & 0.82 & $84.04^{\mathrm{b}}$ & 0.52 & $96 \cdot 07^{c}$ & 1.98 & $177 \cdot 94$ & $<0.001^{\mathrm{ab}, \mathrm{ac}, \mathrm{bc}}$ \\
\hline Fat & $76 \cdot 58^{a}$ & 0.66 & $74 \cdot 84^{a, b}$ & 0.42 & $69 \cdot 68^{\mathrm{c}}$ & 1.61 & $8 \cdot 38$ & $<0.001^{\mathrm{ac}} ; 0.002^{\mathrm{bc}}$ \\
\hline Fibre & $13 \cdot 71^{a}$ & $0 \cdot 24$ & $15 \cdot 00^{\mathrm{b}}$ & $0 \cdot 15$ & $17 \cdot 34^{\mathrm{C}}$ & 0.58 & $20 \cdot 85$ & $<0.001^{\mathrm{ab}, \mathrm{ac}, \mathrm{bc}}$ \\
\hline \multicolumn{9}{|c|}{$\begin{array}{l}\text { Other Foods group sub-categories } \\
\text { (servings/d) ( } n \text { 4867) }\end{array}$} \\
\hline Mostly sugar & $1 \cdot 12^{\mathrm{a}}$ & 0.05 & $0 \cdot 87^{b}$ & 0.03 & $0 \cdot 66^{\mathrm{b}, \mathrm{c}}$ & $0 \cdot 12$ & $11 \cdot 18$ & $<0.001^{\mathrm{ab}, \mathrm{ac}}$ \\
\hline High-salt/fat & $1 \cdot 12^{\mathrm{a}}$ & 0.04 & $0 \cdot 43^{b}$ & 0.02 & $0.03^{\mathrm{C}}$ & 0.09 & $146 \cdot 38$ & $<0 \cdot 001^{\mathrm{ab}, \mathrm{ac}, \mathrm{bc}}$ \\
\hline High-calorie beverages & $1 \cdot 38^{a}$ & 0.04 & $0 \cdot 65^{\mathrm{b}}$ & 0.03 & $-0.03^{c}$ & $0 \cdot 11$ & $133 \cdot 48$ & $<0 \cdot 001^{\mathrm{ab}, \mathrm{ac}, \mathrm{bc}}$ \\
\hline Low-calorie beverages & $1 \cdot 11^{\mathrm{a}}$ & 0.06 & $1 \cdot 28^{a, b}$ & 0.04 & $1 \cdot 81^{\mathrm{C}}$ & $0 \cdot 16$ & $9 \cdot 00$ & $<0.001^{\mathrm{ac}} ; 0.001^{\mathrm{bc}}$ \\
\hline High-sugar/fat & $0 \cdot 74^{\mathrm{a}}$ & 0.03 & $0.59^{b}$ & 0.02 & $0.46^{\mathrm{b}, \mathrm{c}}$ & 0.08 & 9.94 & $<0.001^{\mathrm{ab}} ; 0.001^{\mathrm{ac}}$ \\
\hline
\end{tabular}

a,b,c Mean values within a row with unlike superscript letters were significantly different ( $P$ value for pairwise comparisons given in final column).

${ }^{*}$ Adjusted for total energy intake.

+Univariate analyses.

‡Diet quality: poor $=$ met 0 or 1 food group recommendations, average $=$ met 2 or 3 food group recommendations, superior $=$ met all 4 food group recommendations.

\section{Diet quality and meal behaviours}

MANCOVA used to examine factors associated with meal frequency yielded significant main effects for sex (Wilks' $\lambda=1 \cdot 00, F[4,3705]=3 \cdot 75, P=0 \cdot 005, \eta_{\mathrm{p}}^{2}=0 \cdot 004$ ) and diet quality (Wilks' $\lambda=0 \cdot 98, \quad F[8,7410]=9 \cdot 30$, $\left.P<0 \cdot 001, \eta_{\mathrm{p}}^{2}=0 \cdot 010\right)$, but not for the interaction between diet quality and sex (Wilks' $\lambda=1 \cdot 00, F[8,7410]=1 \cdot 29$, $\left.P=0 \cdot 242, \eta_{\mathrm{p}}^{2}=0 \cdot 001\right)$. Univariate analyses for sex were not significant. Follow-up univariate analyses revealed those with poor diet quality had a significantly lower frequency of consuming breakfast compared with both those with average diet quality as well as those with superior diet quality (Table 4).

The association between diet quality and sex with frequency of consuming meals away from home revealed significant $F$ values (MANCOVA) for sex (Wilks' $\lambda=0 \cdot 98$, $\left.F[6,4193]=11 \cdot 45, P<0 \cdot 001, \eta_{\mathrm{p}}^{2}=0 \cdot 016\right)$ and diet quality $\left(\right.$ Wilks' $\lambda=0 \cdot 98, F[12,8386]=5 \cdot 82, P<0 \cdot 001, \eta_{\mathrm{p}}^{2}=0 \cdot 008$ ), but not for an interaction between diet quality and sex (Wilks' $\lambda=1 \cdot 00, F[12,8386]=0 \cdot 77, P=0 \cdot 687, \eta_{\mathrm{p}}^{2}=0 \cdot 001$ ). Univariate analyses revealed that boys had higher frequencies of consuming meals away from home than girls at all locations: school cafeterias $(F[1,4198]=7 \cdot 36, P=0 \cdot 007$, $\left.\eta_{\mathrm{p}}^{2}=0 \cdot 002\right)$, fast food/take out $(F[1,4198]=27 \cdot 05$, $\left.P<0.001, \eta_{\mathrm{p}}^{2}=0.006\right)$, other restaurants $(F[1,4198]=27 \cdot 93$, $\left.P<0 \cdot 001, \quad \eta_{\mathrm{p}}^{2}=0 \cdot 007\right)$, vending machines $(F[1,4198]=$ 45.51, $\left.P<0 \cdot 001, \eta_{\mathrm{p}}^{2}=0 \cdot 011\right)$, snack bars $(F[1,4198]=$ $\left.31 \cdot 23, P<0 \cdot 001, \eta_{\mathrm{p}}^{2}=0 \cdot 007\right)$ and convenience stores $(F[1$, $\left.4198]=36 \cdot 36, P<0 \cdot 001, \eta_{\mathrm{p}}^{2}=0 \cdot 009\right)$. Follow-up univariate analyses revealed that those with poor diet quality consumed significantly more meals or snacks away from home at all locations compared with those with average and/or superior diet quality (Table 4).

\section{Physical activity}

More than half of the students (57\%) had physical activity levels below a score of 3 (moderate activity), with average physical activity at 2.88 (SD 0.68). Boys (2.99 (SD 0.71)) had significantly higher physical activity levels $(P<0 \cdot 001)$ than girls (2.79 (sD 0.63)), and younger students ( $\leq 13$ years; $2 \cdot 97$ (SD 0.66)) had significantly higher physical activity levels $(P<0 \cdot 001)$ than older students ( $\geq 14$ years; $2 \cdot 81$ (SD 0 68)).

Significant differences were observed in physical activity levels between diet quality groups $(F[2,4391]=47 \cdot 80$, 
Table 4 Group differences in meal behaviours* based on diet qualityt among adolescents aged 11-17 years, Alberta, Canada

\begin{tabular}{|c|c|c|c|c|c|c|c|c|}
\hline & \multicolumn{6}{|c|}{ Diet quality $\ddagger$} & \multirow[b]{3}{*}{$F$ value } & \multirow[b]{3}{*}{$P$ value } \\
\hline & \multicolumn{2}{|c|}{ Poor } & \multicolumn{2}{|c|}{ Average } & \multicolumn{2}{|c|}{ Superior } & & \\
\hline & Mean & SE & Mean & SE & Mean & SE & & \\
\hline \multicolumn{9}{|c|}{ Meal frequency ( $n$ 3718) } \\
\hline Breakfast & $4 \cdot 20^{a}$ & 0.04 & $4 \cdot 34^{\mathrm{b}}$ & 0.03 & $4 \cdot 69^{\mathrm{c}}$ & $0 \cdot 13$ & $8 \cdot 46$ & $0.007^{\mathrm{ab}} ;<0.001^{\mathrm{ac}} ; 0.006^{\mathrm{bc}}$ \\
\hline Lunch & $4 \cdot 66$ & 0.03 & $4 \cdot 69$ & 0.02 & $4 \cdot 86$ & 0.08 & $2 \cdot 78$ & NSD \\
\hline Dinner & $4 \cdot 90$ & 0.02 & $4 \cdot 88$ & 0.01 & 4.94 & 0.05 & $0 \cdot 80$ & NSD \\
\hline Snacks & $3 \cdot 52$ & 0.04 & $3 \cdot 46$ & 0.03 & $3 \cdot 37$ & $0 \cdot 11$ & $1 \cdot 41$ & NSD \\
\hline \multicolumn{9}{|c|}{$\begin{array}{l}\text { Consuming meals and snacks away } \\
\text { from homell ( } n \text { 4205) }\end{array}$} \\
\hline School cafeteria & $2 \cdot 43^{a}$ & 0.04 & $2 \cdot 26^{b}$ & 0.03 & $2 \cdot 21$ & 0.09 & 4.96 & $0.002^{\mathrm{ab}}$ \\
\hline Fast food/take out & $2 \cdot 37^{\mathrm{a}}$ & 0.03 & $2 \cdot 25^{\mathrm{b}}$ & 0.02 & $2 \cdot 08^{c}$ & 0.06 & $10 \cdot 18$ & $0.001^{\mathrm{ab}} ;<0.001^{\mathrm{ac}} ; 0.004^{\mathrm{bc}}$ \\
\hline Other restaurants & $2 \cdot 10^{\mathrm{a}}$ & 0.02 & $2 \cdot 02$ & 0.02 & $1 \cdot 91^{b}$ & 0.05 & $5 \cdot 31$ & $0.002^{\mathrm{ab}}$ \\
\hline Vending machines & $2 \cdot 31^{a}$ & 0.03 & $2 \cdot 08^{\mathrm{b}}$ & 0.03 & $1 \cdot 83^{\mathrm{c}}$ & 0.07 & $19 \cdot 20$ & $<0.001^{\mathrm{ab}, \mathrm{ac}} ; 0.001^{\mathrm{bc}}$ \\
\hline Snack bars & $2 \cdot 33^{a}$ & 0.03 & $2 \cdot 15^{\mathrm{b}}$ & 0.03 & $1 \cdot 93^{c}$ & 0.07 & $12 \cdot 91$ & $<0.001^{\mathrm{ab}, \mathrm{ac}} ; 0.002^{\mathrm{bc}}$ \\
\hline Convenience stores & $2 \cdot 66^{\mathrm{a}}$ & 0.03 & $2 \cdot 42^{b}$ & 0.02 & $2 \cdot 11^{\mathrm{c}}$ & 0.07 & $26 \cdot 55$ & $<0.001^{\mathrm{ab}, \mathrm{ac}, \mathrm{bc}}$ \\
\hline
\end{tabular}

NSD, no significant difference.

a,b,c Mean values within a row with unlike superscript letters were significantly different ( $P$ value for pairwise comparisons given in final column).

${ }^{*}$ Adjusted for total energy intake.

tUnivariate analyses.

fDiet quality: poor = met 0 or 1 food group recommendations, average $=$ met 2 or 3 food group recommendations, superior $=$ met all 4 food group recommendations.

$\S$ Meal frequency: never $=1$, on weekends only $=2$, less than half of the week (three or fewer days each week) $=3$, more than half of the week (four or more days each week) $=4$, every day $=5$; snacks were averaged before analysis (morning snacks, afternoon snacks, evening snacks).

IIConsuming meals and snacks away from home: rarely or never $=1$, once a month $=2$, once a week $=3,2-6$ times a week $=4$, once a day $=5$.

$\left.P<0 \cdot 001, \eta_{\mathrm{p}}^{2}=0 \cdot 021\right)$ based on ANOVA. Students with poor diet quality $(2 \cdot 80(\mathrm{SD} 0 \cdot 02))$ had significantly lower levels of physical activity compared with those with both average (2.91 (SD 0.01), $P<0 \cdot 001$ ) and superior diet quality (3.16 (SD 0·04), $P<0 \cdot 001$ ). Further, students with average diet quality had significantly lower levels of physical activity than their peers with superior diet quality $(P<0 \cdot 001)$.

\section{Discussion}

The purpose of the present study was to use Web-SPAN to assess overall diet quality, and evaluate the relationship between diet quality, nutrient intakes, meal behaviours (i.e. meal skipping and consuming meals away from home) and physical activity levels. Although numerous studies have examined nutrition and physical activity status of adolescents, few have used a measure of foodbased diet quality to examine these associations.

Macronutrient, micronutrient and CFGHE servings were assessed using data from the $24 \mathrm{~h}$ recall component of Web-SPAN. In comparison to recommendations as well as differences observed between sex, both boys' and girls' intakes were consistent with previous reports ${ }^{(2)}$ and were within the AMDR. Consistent with previous studies ${ }^{(37,38)}$, micronutrient intakes were low based on a high percentage of students below the EAR or adjusted average intakes below the AI. Older boys and girls (14-17 years) had a greater risk of inadequacy than younger boys and girls (9-13 years) for most nutrients. This indicates that although micronutrient requirements increase with age, older adolescents had similar intakes to their younger peers and were not consuming optimal nutritional intakes for their physiological requirements. Adequate consumption of micronutrients is necessary for prevention of nutrient deficiency and reduction of chronic disease ${ }^{(22-26)}$. Given the observed low levels of $\mathrm{Zn}, \mathrm{Ca}$ and fibre, many students reported suboptimal nutritional intakes that could have an effect on long-term health and onset of disease.

Intakes of foods found in CFGHE were low for both boys and girls. Although boys were more likely to meet CFGHE recommendations for all four food groups than girls, less than $50 \%$ of boys met the recommendations for Vegetables and Fruit and Milk Products. Among girls intakes were even poorer, with less than $50 \%$ meeting the recommendations for all food groups. These results are consistent with the micronutrient analyses, where inadequate consumption of $\mathrm{Zn}, \mathrm{Ca}$ and fibre correspond to low intakes of Meats and Alternatives, Milk Products, and Grain Products and Vegetables and Fruits, respectively. Given the observed high intakes of Other Foods, it is likely students were compensating for low CFGHE intakes with Other Foods, which concurrently resulted in low micronutrient intakes. This finding is supported by the 2004 Canadian Community Health Survey (CCHS), which reported Other Foods as the second largest contributor of total energy in the diet of adolescents ${ }^{(2)}$. Furthermore, a study by Jacobs Starkey et al. ${ }^{(39)}$ found that Other Foods provided more than 25\% of energy among adolescents aged 13-17 years, making Other Foods the predominant source of energy.

Overall adherence to CFGHE was low; only 8\% of students were estimated as having superior diet quality. More boys than girls reported having superior diet quality. 
Although each food group is individually important, total health and wellness relies on the appropriate balance and variety of all food groups ${ }^{(4)}$. The low proportion of students with superior diet quality reflects the high prevalence of micronutrient inadequacy, and supports the need for improved adherence to CFGHE. Further, significant differences in macronutrient and Other Foods intake existed between those with average and superior diet quality which revealed a step-wise improvement in nutrition when diet quality increased from poor to average to superior. Mean differences in nutrient intakes between diet quality groups were small, indicating that although trends were observed, the biological relevance of consuming small, yet significant differences in macronutrients is unclear.

Breakfast skipping is highly prevalent, and is associated with poor nutritional behaviours ${ }^{(8,40,41)}$ and increased prevalence of overweight ${ }^{(40)}$. The 2004 CCHS revealed that $10 \%$ of adolescents reported skipping breakfast the day before they were interviewed ${ }^{(2)}$. However, less is understood regarding the association between diet quality and meal behaviours (i.e. meal skipping and consuming meals away from home). In the current study, those with poor diet quality had a lower frequency of breakfast consumption than those with average and superior diet quality. This suggests consumption of breakfast is an important determinant of a student's ability to meet CFGHE and DRI recommendations. As expected, increased frequency of consuming meals or snacks away from home was associated with lower diet quality scores. Fast-food consumption has been previously associated with higher percentage of energy from fat, servings of soft drinks, and lower intakes of fruit, vegetables and milk ${ }^{(42)}$. Although suboptimal nutritional intakes have been previously associated with fast-food consumption, less is known regarding the consumption of meals at additional locations. Results from our study indicated students were more likely to have lower diet quality scores when they consumed meals or snacks at any location away from home.

Consistent with previous reports ${ }^{(1,43)}$, more than half of the students $(57 \%)$ had physical activity levels below a score of 3 on the PAQ-C and boys had higher levels of physical activity than girls. In comparison with a study done by Kowalski et $a l^{(32)}$, physical activity levels were slightly lower in the current study. However, the difference is likely due to the age of the participants in each study, as it is known that physical activity decreases with age $^{(3,43)}$. In the current study the age range was $11-17$ years, while students who participated in the study by Kowalski et al. ranged in age from 8 to 13 years. Students with poor diet quality had significantly lower levels of physical activity than those with both average and superior diet quality. In addition, students with average diet quality had significantly lower levels of physical activity than their peers with superior diet quality. Similar to the improved nutrition result, this also suggests a step- wise improvement in physical activity when diet quality increased from poor to average to superior; thus, students with the highest activity levels also had the highest diet quality scores. This suggests a clustering of positive lifestyle behaviours, which is consistent with previous reports from both Canada ${ }^{(41)}$ and the USA ${ }^{(6,44,45)}$. Although socioeconomic status (SES) was not measured in the current study, results from a previous web-based survey indicated vegetable and fruit, fibre and breakfast consumption increased with increasing income, and that consumption of added sugar decreased $^{(13)}$. Further, Minaker et al. reported that students attending private schools had a higher consumption of fibre and a lower consumption of sweetened drinks compared with their peers attending public school $^{(13)}$. Similarly, adolescents living in lower-SES neighbourhoods are less physically active and at greater risk of being overweight ${ }^{(46,47)}$. Thus, the association between diet quality and physical activity observed in the current study may be partially explained by household and neighbourhood SES of students.

Limitations of our study include the cross-sectional design and the use of self-reported survey data. The cross-sectional design prevents the assessment of temporal causality. With regard to self-reports, although validated survey components were used, many adolescents experience recall bias, response bias and underreporting of energy intake ${ }^{(48,49)}$. Additional limitations include the possibility of seasonal variation in nutrient intakes as well as the clustering of students within schools, which was not examined during statistical analyses. However, strengths include anonymity which is an important determinant when adolescents choose to report honest answers ${ }^{(50)}$, decreased reactivity as students were not aware when the survey was to be completed, and the large sample size.

\section{Conclusions}

In summary, our sample of Alberta adolescents met the AMDR for macronutrients. However, adolescents consumed suboptimal intakes of micronutrients in addition to low intakes of CFGHE foods and high intakes of Other Foods. The high number of students with poor diet quality reflects these intakes, meal skipping and increased meals away from home. In addition, students with high activity levels also had high diet quality scores, which suggest a clustering of positive lifestyle behaviours. Further research in this sample of adolescents is necessary to explain reasons for clustering of behaviours as well as the differences observed between boys and girls in terms of physical activity and nutrient intakes. Consequently, this research on the clustering, age trends and sex differences of chronic disease-related risk factors in this sample has subsequently been completed ${ }^{(11)}$. Improvements in nutrition and physical activity were observed in 
a step-wise fashion when diet quality increased from poor to average to superior. It is recommended that suboptimal health behaviours be targeted in early adolescence, as even small improvements in diet quality and physical activity may result in a greater frequency of optimal health behaviours and could have a lasting impact on long-term health.

\section{Acknowledgements}

This research was supported by the Alberta Heritage Foundation for Medical Research (AHFMR) and the Canadian Institutes of Health Research/Heart and Stroke Foundation (CIHR/HSF). K.E.S. received funding from the Danone Institute of Canada (Doctoral Student Award), CIHR/HSF and AHFMR Health Research Studentship. R.C.P. and K.D.R. acknowledge Salary Awards from AHFMR and CIHR. The authors declare that they have no competing interests. K.E.S. contributed to the design of the study and the development of Web-SPAN; coordinated the study including recruitment, data collection and data analysis; and wrote the manuscript. L.E.F. provided assistance in recruitment and data collection. S.N.F. contributed to data analysis and interpretation. J.C.S., R.C.P., K.D.R. and L.J.M. contributed to the design of the study and the development of Web-SPAN. R.M.H. developed the original web-survey tool, and assisted in the development of Web-SPAN. L.J.M. supervised the entire study. All authors edited and approved the manuscript.

\section{References}

1. Shields M (2005) Nutrition: Findings from the Canadian Community Health Survey. Measured Obesity: Overweight Canadian children and adolescents. http://www.statcan.ca/ english/research/82-620-MIE/2005001/pdf/cobesity.pdf (accessed August 2008).

2. Garriguet D (2004) Nutrition: Findings from the Canadian Community Health Survey. Overview of Canadians' eating habits. http://www.statcan.ca/english/research/82-620MIE/82-620-MIE2006002.pdf (accessed August 2008).

3. Canadian Fitness and Lifestyle Research Institute (2006) 2005 Physical Activity and Sport Monitor. http://www. cflri.ca/eng/statistics/surveys/pam2005.php (accessed August 2008).

4. Health Canada (1992) Canada's Food Guide to Healthy Eating. http://www.hc-sc.gc.ca/fn-an/alt_formats/hpfb-dgpsa/ pdf/food-guide-aliment/fg_rainbow-arc_en_ciel_ga_e.pdf (accessed August 2008).

5. Health Canada and the Canadian Society for Exercise Physiology (2002) Canada's Physical Activity Guide for Youth. http://www.phac-aspc.gc.ca/pau-uap/paguide/ child_youth/pdf/guide_y_e.pdf (accessed August 2008).

6. Sanchez A, Norman GJ, Sallis JF, Calfas KJ, Cella J \& Patrick K (2007) Patterns and correlates of physical activity and nutrition behaviors in adolescents. Am J Prev Med 32, 124-130.

7. Raine KD (2004) Overweight and obesity in Canada: A population health perspective. http://www.cihi.ca/ cihiweb/dispPage.jsp?cw_page=GR_1130_E (accessed August 2008).
8. Woodruff SJ, Hanning RM, Lambraki I, Storey KE \& McCargar L (2008) Healthy Eating Index-C is compromised among adolescents with body weight concerns, weight loss dieting, and meal skipping. Body Image 5, 404-408.

9. Hurley KM, Oberlander SE, Merry BC, Wrobleski MM, Klassen AC \& Black MM (2009) The healthy eating index and youth healthy eating index are unique, nonredundant measures of diet quality among low-income, African American adolescents. J Nutr 139, 359-364.

10. Dietz WH (1998) Health consequences of obesity in youth: childhood predictors of adult disease. Pediatrics $\mathbf{1 0 1}$, 518-525.

11. Plotnikoff RC, Karunamuni N, Spence JC, Storey K, Forbes L, Raine K, Wild TC \& McCargar L (2009) Chronic diseaserelated lifestyle risk factors in a sample of Canadian adolescents. J Adolesc Health (In the Press).

12. Hanning RM, Woodruff SJ, Lambraki I, Jessup L, Driezen P \& Murphy CC (2007) Nutrient intakes and food consumption patterns among Ontario students in grades six, seven, and eight. Can J Public Health 98, 12-16.

13. Minaker LM, McCargar L, Lambraki I, Jessup L, Driezen P, Calengor K \& Hanning RM (2006) School region socioeconomic status and geographic locale is associated with food behaviour of Ontario and Alberta adolescents. Can J Public Health 97, 357-361.

14. Rockett HRH \& Colditz GA (1997) Assessing diets of children and adolescents. Am J Clin Nutr 65, 4 Suppl., 1116S-1122S.

15. Calengor KE (2007) Nutrition, physical activity and related health behaviours in youth as assessed by a web-survey. PhD Thesis, University of Alberta.

16. Lowik MR, Hulshof KF \& Brussaard JH (1999) Food-based dietary guidelines: some assumptions tested for The Netherlands. Br J Nutr 81, Suppl, 2, S143-S149.

17. Waijers PM, Feskens EJ \& Ocke MC (2007) A critical review of predefined diet quality scores. Br J Nutr $\mathbf{9 7}$, 219-231.

18. Health Canada (2001) Canadian Nutrient File. http://www. hc-sc.gc.ca/food-aliment/ns-sc/nr-rn/surveillance/cnf-fcen/ e_index.html (accessed August 2008).

19. Health Canada (2007) Eating Well with Canada's Food Guide. http://www.hc-sc.gc.ca/fn-an/alt_formats/hpfb-dgpsa/ pdf/food-guide-aliment/view_eatwell_vue_bienmang_e.pdf (accessed August 2008).

20. Alberta Learning (2002) Health and life skills, kindergarten to grade 9. http://www.education.gov.ab.ca/k_12/curriculum/ bySubject/healthpls/health.pdf (accessed December 2006).

21. Alberta Learning (2005) Career and technology studies. http://www.education.gov.ab.ca/k\%5F12/curriculum/by Subject/cts/foods/fodpos.pdf (accessed December 2006).

22. Institute of Medicine (2005) Dietary Reference Intakes for Energy, Carbohydrates, Fiber, Fat, Fatty Acids, Cholesterol, Protein, and Amino Acids (Macronutrients). Washington, DC: National Academy Press.

23. Institute of Medicine (2000) Dietary Reference Intakes for Vitamin C, Vitamin E, Selenium, and Carotenoids. Washington, DC: National Academy Press.

24. Institute of Medicine (2000) Dietary Reference Intakes for Vitamin A, Vitamin K, Arsenic, Boron, Chromium, Copper, Iodine, Iron, Manganese, Molybdenum, Nickel, Silicon, Vanadium, and Zinc. Washington, DC: National Academy Press.

25. Institute of Medicine (1998) Dietary Reference Intakes for Thiamin, Riboflavin, Niacin, Vitamin $B_{6}$, Folate, Vitamin $B_{12}$, Pantothenic Acid, Biotin, and Choline. Washington, DC: National Academy Press.

26. Institute of Medicine (1997) Dietary Reference Intakes for Calcium, Phosphorus, Magnesium, Vitamin D, and Fluoride. Washington, DC: National Academy Press. 
27. Institute of Medicine (2000) Dietary Reference Intakes: Applications in Dietary Assessment. Washington, DC: National Academy Press.

28. Neumark-Sztainer D, Wall MM, Story M \& Perry CL (2003) Correlates of unhealthy weight-control behaviors among adolescents: implications for prevention programs. Health Psychol 22, 88-98.

29. Neumark-Sztainer D, Story M, Hannan PJ, Perry CL \& Irving LM (2002) Weight-related concerns and behaviors among overweight and nonoverweight adolescents: implications for preventing weight-related disorders. Arch Pediatr Adolesc Med 156, 171-178.

30. Kowalski KC, Crocker PRE \& Donen RM (2004) The Physical Activity Questionnaire for Older Children (PAQ-C) and Adolescents (PAQ-A) Manual. http://hkin.educ.ubc.ca/ behavioural/PAQ\%20manual.pdf (accessed August 2008).

31. Crocker PRE, Bailey DA, Faulkner RA, Kowalski KC \& McGrath R (1997) Measuring general levels of physical activity: preliminary evidence for the Physical Activity Questionnaire for Older Children. Med Sci Sports Exerc 29, 1344-1349.

32. Kowalski KC, Crocker PRE \& Faulkner RA (1997) Validation of the Physical Activity Questionnaire for Older Children. Pediatr Exerc Sci 9, 174-186.

33. Barr SI (2006) Applications of Dietary Reference Intakes in dietary assessment and planning. Appl Pbysiol Nutr Metab 31, 66-73.

34. Nusser SM, Carriquiry AL, Dodd KW \& Fuller WA (1996) A semiparametric transformation approach to estimating usual daily intake distributions. J Am Stat Assoc 91, 1440-1449.

35. Tabachnick BG \& Fidell LS (2007) Using Multivariate Statistics, 5th ed. New York: Pearson Education, Inc.

36. D'Alonzo KT (2004) The Johnson-Neyman procedure as an alternative to ANCOVA. West I Nurs Res 26, 804-812.

37. Gray-Donald K, Jacobs-Starkey L \& Johnson-Down L (2000) Food habits of Canadians: reduction in fat intake over a generation. Can J Public Health 91, 381-385.

38. Veugelers PJ, Fitzgerald AL \& Johnston E (2005) Dietary intake and risk factors for poor diet quality among children in Nova Scotia. Can J Public Health 96, 212-216.

39. Jacobs Starkey L, Johnson-Down L \& Gray-Donald K (2001) Food habits of Canadians: comparison of intakes in adults and adolescents to Canada's food guide to healthy eating. Can J Diet Pract Res 62, 61-69.

40. Rampersaud GC, Pereira MA, Girard BL, Adams J \& Metzl JD (2005) Breakfast habits, nutritional status, body weight, and academic performance in children and adolescents. $J$ Am Diet Assoc 105, 743-760.

41. Cohen B, Evers S, Manske S, Bercovitz K \& Edward HG (2003) Smoking, physical activity and breakfast consumption among secondary school students in a southwestern Ontario community. Can J Public Health 94, 41-44.

42. French SA, Story M, Neumark-Sztainer D, Fulkerson JA \& Hannan P (2001) Fast food restaurant use among adolescents: associations with nutrient intake, food choices and behavioral and psychosocial variables. Int $J$ Obes Relat Metab Disord 25, 1823-1833

43. Canadian Fitness and Lifestyle Research Institute (2004) 2004 Physical Activity and Sport Monitor. http:// www.cflri.ca/eng/statistics/surveys/pam2004.php (accessed August 2008).

44. Pronk NP, Anderson LH, Crain AL, Martinson BC, O'Connor PJ, Sherwood NE \& Whitebird RR (2004) Meeting recommendations for multiple healthy lifestyle factors. Prevalence, clustering, and predictors among adolescent, adult, and senior health plan members. Am J Prev Med 27, 25-33.

45. Lytle LA, Kelder SH, Perry CL \& Klepp K-I (1995) Covariance of adolescent health behaviours: the Class of 1989 study. Health Educ Res 10, 133-146.

46. Janssen I, Boyce WF, Simpson K \& Pickett W (2006) Influence of individual- and area-level measures of socioeconomic status on obesity, unhealthy eating, and physical inactivity in Canadian adolescents. Am J Clin Nutr 83, 139-145.

47. Gordon-Larsen P, Nelson MC, Page P \& Popkin BM (2006) Inequality in the built environment underlies key health disparities in physical activity and obesity. Pediatrics 117, 417-424.

48. Bandini LG, Must A, Cyr H, Anderson SE, Spadano JL \& Dietz WH (2003) Longitudinal changes in the accuracy of reported energy intake in girls $10-15$ y of age. Am J Clin Nutr 78, 480-484.

49. Livingstone MB, Prentice AM, Coward WA, Strain JJ, Black AE, Davies PSW, Stewart CM, McKenna PG \& Whitehead RG (1992) Validation of estimates of energy intake by weighed dietary record and diet history in children and adolescents. Am J Clin Nutr 56, 29-35.

50. Supple AJ, Aquilino WS \& Wright DL (1999) Collecting sensitive self-report data with laptop computers: impact on the response tendencies of adolescents in a home interview. J Res Adolesc 9, 467-488. 